\title{
Lisdexamfetamine Dimesylate Effects on the Pharmacokinetics of Cytochrome P450 Substrates in Healthy Adults in an Open-Label, Randomized, Crossover Study
}

\author{
James Ermer ${ }^{1} \cdot$ Mary Corcoran $^{1} \cdot$ Patrick Martin $^{1}$
}

Published online: 11 April 2015

(C) The Author(s) 2015. This article is published with open access at Springerlink.com

\begin{abstract}
Introduction This open-label, randomized, two-period drug interaction study assessed lisdexamfetamine dimesylate (LDX) effects on cytochrome P450 (CYP) enzyme (CYP1A2, CYP2D6, CYP2C19, and CYP3A) activity.

Methods Thirty healthy volunteers were administered the Cooperstown cocktail (CYP1A2 [caffeine $200 \mathrm{mg}$ ], CYP2D6 [dextromethorphan $30 \mathrm{mg}$ ], CYP2C19 [omeprazole $40 \mathrm{mg}$ ], and CYP3A [midazolam $0.025 \mathrm{mg} / \mathrm{kg}$ ] substrates) or Cooperstown cocktail + oral LDX $70 \mathrm{mg}$. Blood samples for pharmacokinetic analysis were collected pre-dose and serially for $72 \mathrm{~h}$ post-dose. Treatment differences in the primary endpoints, maximum plasma concentration $\left(C_{\max }\right)$ and area under the plasma concentration versus time curve from 0 to infinity $\left(\mathrm{AUC}_{0-\infty}\right)$, were assessed using geometric mean ratios with $90 \%$ CIs.

Results Geometric least squares (LS) means (without versus with $\mathrm{LDX})$ for $C_{\max }(\mathrm{ng} / \mathrm{mL})$ were 5370 versus 5246 for caffeine, 2.43 versus 2.87 for dextromethorphan, 35.23 versus 35.11 for midazolam, and 677.9 versus 466.9 for omeprazole; and for $\mathrm{AUC}_{0-\infty}(\mathrm{ng} \cdot \mathrm{h} / \mathrm{mL})$ were 56,207 versus 56,688 for caffeine, 34.85 versus 37.27 for dextromethorphan, 92.07 versus 93.04 for midazolam, and 1428 versus 1499 for omeprazole. Geometric LS mean ratios were within the standard bioequivalence testing
\end{abstract}

Electronic supplementary material The online version of this article (doi:10.1007/s40268-015-0090-z) contains supplementary material, which is available to authorized users.

James Ermer

jaermer@shire.com

1 Shire, 725 Chesterbrook Blvd, Wayne, PA 19087, USA range, except for omeprazole and dextromethorphan $C_{\max }$. Parent/metabolite $C_{\max }$ and $\mathrm{AUC}_{0-\infty}$ ratios were similar between treatments except for dextromethorphan/dextrorphan $\mathrm{AUC}_{0-\infty}$ ratio, which was lower with LDX. No serious or severe treatment-emergent adverse events were reported.

Conclusions LDX did not alter CYP1A2, CYP2D6, or CYP3A activity. A small $C_{\max }$ reduction for omeprazole and its metabolite was observed, possibly reflecting an effect either on the activity of CYP2C19 or omeprazole absorption.

\section{Key Points}

Lisdexamfetamine did not alter the activity of the CYP1A2, CYP2D6, or CYP3A enzymes in healthy volunteers.

A small $C_{\max }$ reduction for omeprazole and its metabolite was observed, possibly reflecting an effect either on the activity of CYP2C19 or omeprazole absorption.

\section{Introduction}

Individuals with attention-deficit/hyperactivity disorder (ADHD) have high rates of comorbid psychiatric disorders, including anxiety, major depressive disorder, and disruptive disorders [1-3]. To treat these psychiatric comorbidities, individuals with ADHD may be prescribed additional pharmacotherapy. 
In the presence of polypharmacy, there is a potential for drug-drug interactions. Many of the medications used to treat these comorbid psychiatric disorders (e.g., antidepressants for mood disorders) are known to interact with cytochrome P450 (CYP) enzymes [4]. For instance, fluoxetine strongly inhibits CYP2D6, CYP1A2, and CYP2C19 activity; similarly, paroxetine strongly inhibits CYP2D6 activity and nefazodone potently inhibits CYP3A4 activity [4]. In addition, moderate inhibition of other CYP enzymes (e.g., CYP2D6, CYP2C9, and CYP3A4) has been reported with bupropion, duloxetine, fluoxetine, and fluvoxamine [4].

Psychostimulants, such as amphetamines, are used in the treatment of ADHD [5]. Because it has been reported that D-amphetamine is metabolized by CYP2D6 [6], there could be competition for this isozyme when it is administered with other CYP2D6 substrates. Therefore, it is important to determine the effects of stimulant medications on the pharmacokinetic profiles of selected CYP substrates to determine if there is a potential for drug-drug interactions.

Lisdexamfetamine dimesylate (LDX), a prodrug of D-amphetamine [7], is approved in the United States to treat patients 6 years and older with ADHD and adults with moderate to severe binge eating disorder [8]. Absorption of LDX occurs primarily via carrier-mediated transport in the small intestine [7]. LDX is then metabolized in red blood cells into D-amphetamine and L-lysine [7].

In an in vitro study in human liver microsomes, LDX did not produce concentration-dependent or mechanism-based inhibition of several CYP isozymes, including CYP1A2, CYP2C19, CYP2D6, and CYP3A4 [9]. Potential interactions of LDX with medications metabolized by CYP enzymes have also been examined in two clinical trials in healthy volunteers $[10,11]$. In one open-label study, LDX administered in combination with venlafaxine extended release (VXR), which is metabolized by CYP2D6 and CYP3A4 [4], was associated with small increases in VXR exposure, small decreases in exposure to the primary VXR metabolite ( $O$-desmethylvenlafaxine [ODV]), and no change in composite VXR + ODV exposure when compared with VXR alone in healthy volunteers [10]. When LDX was administered to healthy volunteers in combination with guanfacine extended release (GXR), which is metabolized by CYP3A4 [12], there were no changes in LDX or D-amphetamine exposure when compared with LDX alone. Although a small increase in GXR maximum plasma concentration $\left(C_{\max }\right)$ was observed with the LDX + GXR combination compared with GXR alone, this change was not considered clinically meaningful [11]. Although these findings suggest there may be only limited interactions of LDX with CYP2D6 and CYP3A4 enzyme activity, they are based on relatively few studies so a more comprehensive analysis of the effects of LDX on CYP substrates would provide more insight into the potential for drug-drug interactions with medications metabolized by these systems.

The primary objective of the current study was to assess the pharmacokinetic profiles of substrates of the CYP1A2 (caffeine), CYP2D6 (dextromethorphan), CYP2C19 (omeprazole), and CYP3A (midazolam) enzymes when administered as the Cooperstown cocktail alone or in combination with LDX. A four-drug Cooperstown cocktail was used to identify potential drug interactions because it offers the advantages of using commercially available drugs that have low adverse event (AE) profiles and that are specific for selected CYP enzymes [13]. In addition, a secondary objective was to provide tolerability and safety data for LDX when administered in combination with the CYP enzyme substrates.

\section{Materials and Methods}

\subsection{Study Design and Treatment}

The protocol was approved by the institutional review board of the study site before the initiation of the study, and the study was conducted in accordance with the International Conference on Harmonisation and Good Clinical Practice and with the Declaration of Helsinki. All participants provided written informed consent in accordance with Good Clinical Practice guidelines.

This open-label, randomized, two-period, drug-drug interaction study was conducted by Clinical Pharmacology of Miami, Inc (Miami, FL, USA) between December 21, 2012 and February 4, 2013 and consisted of a 28-day screening phase and two single-dose, 5-day treatment periods separated by a 7-day washout period between doses. Before the first treatment period, participants were randomized in a 1:1 ratio to a single administration of either the Cooperstown cocktail alone, which included substrates for the CYP1A2 (caffeine), CYP2D6 (dextromethorphan), CYP2C19 (omeprazole), and CYP3A (midazolam) enzymes, or the Cooperstown cocktail in combination with $70 \mathrm{mg}$ LDX. The alternate treatment was administered during the second treatment period. Randomization occurred immediately before dosing on day 1 of the first treatment period and was accomplished by assigning a 4-digit randomization number to each participant; the randomization schedule was produced and held by PRA International (Lenexa, KS, USA).

Caffeine $(200 \mathrm{mg})$, dextromethorphan $(30 \mathrm{mg})$, and omeprazole $(40 \mathrm{mg})$ were each administered orally with $240 \mathrm{~mL}$ of room temperature water. On days when LDX was administered, it was also given orally with $240 \mathrm{~mL}$ of room temperature water after omeprazole. All orally administered agents were given within a 1-min period, were 
swallowed whole, and administration was confirmed by mouth check. Midazolam $(0.025 \mathrm{mg} / \mathrm{kg})$ was administered intravenously over a 1-min period within $3 \mathrm{~min}$ of the last orally administered agent; midazolam administration was immediately followed with a $5-\mathrm{mL}$ normal saline intravenous infusion to flush the indwelling catheter.

All participants were required to fast for approximately $10 \mathrm{~h}$ before dosing and for another $4 \mathrm{~h}$ after dosing. Participants were also required to refrain from taking any fluids $4 \mathrm{~h}$ before and $2 \mathrm{~h}$ after dosing and were not permitted to lie down for the first $4 \mathrm{~h}$ following administration. A follow-up telephone contact was made approximately 1 week after the final day of the last treatment period to identify ongoing and/or new AEs and concomitant medications taken since discharge.

\subsection{Participants}

Eligible participants were healthy men or nonpregnant, nonlactating women who were $18-45$ years of age, had a body mass index of $18.5-30.0 \mathrm{~kg} / \mathrm{m}^{2}$ at the screening visit, had hemoglobin values $\geq 12 \mathrm{~g} / \mathrm{dL}$ at screening and at the start of treatment, and had no clinically significant or relevant medical history, physical examination, vital signs, electrocardiogram (ECG), or laboratory evaluations. In addition, all participants had the ability to understand and fully comply with the study procedures and to provide consent.

Participants were excluded from study participation if they had a current or recurrent comorbid disease that could affect either the pharmacokinetics of the study drug or the clinical/laboratory assessments or if they had a history of or a current medical/psychiatric disorder that required treatment, made them unlikely to fully comply with the study requirements, or that presented undue risk from the study drug or procedures. Participants were also excluded if they had current or a history of significant cardiac problems, including structural abnormalities, conduction problems, exercise-related events, clinically significant bradycardia, cardiomyopathy, transient ischemic attacks, strokes, or other problems; hypertension, a resting sitting systolic blood pressure (SBP) $>139 \mathrm{mmHg}$, or a diastolic blood pressure (DBP) $>89 \mathrm{mmHg}$; a family history of sudden cardiac death or ventricular arrhythmia; a risk for suicide/suicide ideation or previous suicide attempts; intolerance or hypersensitivity to the study drugs; a history of alcohol or substance abuse within the past year or consumed excessive amounts of alcohol ( $>3$ units/day for men; $>2$ units/day for women); consumed tobacco, another investigational study drug, or substantially changed their eating habits within 30 days of the first study dose; routinely consumed caffeine ( $>2$ units/day) or were prone to caffeine withdrawal headaches; donated blood within
60 days of the start of the study; or were unable to fast or follow standardized diet and meal schedules.

\subsection{Pharmacokinetic Endpoints}

Blood samples for pharmacokinetic analysis were collected pre-dose and serially for $72 \mathrm{~h}$ post-dose. The primary endpoints were $C_{\max }$ and area under the plasma concentration versus time curve from 0 to infinity $\left(\mathrm{AUC}_{0-\infty}\right)$ for the parent compounds (caffeine, dextromethorphan, omeprazole, midazolam) and their metabolites (paraxanthine, dextrorphan, 5-hydroxyomeprazole, 1-hydroxymidazolam) when administered in the Cooperstown cocktail alone or in combination with LDX. Other pharmacokinetic parameters calculated included time of maximum observed concentration sampled during dosing interval $\left(t_{\max }\right)$, terminal half-life $\left(t_{1 / 2}\right)$, total body clearance for extravascular administration divided by the fraction of dose absorbed $(\mathrm{CL} / F)$, and volume of distribution associated with the terminal slope following extravascular administration divided by the fraction of dose absorbed $\left(V_{\mathrm{z}} / F\right)$. In addition, the parent-to-metabolite ratios for $C_{\max }$ and $\mathrm{AUC}_{0-\infty}$ for each CYP substrate were calculated for each treatment regimen, and pharmacokinetic parameters for LDX and D-amphetamine were assessed.

\subsection{Bioanalytical Assays}

\subsubsection{Cytochrome P450 Substrate Analysis}

Concentrations of omeprazole and 5-hydroxyomeprazole, midazolam and 1-hydroxymidazolam, dextromethorphan and dextrorphan, and caffeine and paraxanthine were determined using validated liquid chromatography-tandem mass spectrometry (LC-MS/MS) methods. The LC-MS/MS system was a Sciex API-4000 mass spectrometer (AB SCIEX, Framingham, MA) for omeprazole, 5-hydroxyomeprazole, midazolam, $1^{\prime}$-hydroxymidazolam, caffeine, and paraxanthine or Sciex API-5000 mass spectrometer for dextromethorphan and dextrorphan coupled with a Shimadzu high-performance liquid chromatography (HPLC) system (Shimadzu, Kyoto, Japan).

For omeprazole and 5-hydroxyomeprazole, a $50 \mu \mathrm{L}$ plasma sample was combined with $50 \mu \mathrm{L}$ of a deuterated internal standard (omeprazole-d3 and 5-hydroxyomeprazole-d3 dissolved in 50:50 methanol:water) followed by addition of $600 \mu \mathrm{L}$ acetonitrile to precipitate the protein. After centrifugation, the supernatant was diluted with reconstitution solution (50:50 methanol:water) and $10 \mu \mathrm{L}$ of the sample was injected to the LC-MS/MS system. Chromatographic separation was achieved on a Phenomenex Synergi Hydro-RP $80 \AA 4 \mu \mathrm{m}, 2.0 \times 50 \mathrm{~mm}$ HPLC column (Phenomenex, Torrance, CA) with a mobile phase gradient. 
For midazolam and 1'-hydroxymidazolam, a $200 \mu \mathrm{L}$ plasma sample was combined with $20 \mu \mathrm{L}$ of a deuterated internal standard (midazolam-d4 and $\alpha$-hydroxymidazolam-d4 dissolved in 50:50 methanol:water containing $0.01 \mathrm{~N} \mathrm{HCl}$ ) followed by addition of $1 \mathrm{~mL}$ methanol to precipitate the protein. After centrifugation, the supernatant was diluted with a reconstitution solution $(1 \mathrm{mM}$ ammonium acetate) and $20 \mu \mathrm{L}$ of the sample was injected to the LC-MS/MS system. Chromatographic separation was achieved on an Atlantis T3 $3 \mu \mathrm{m}, 2.1 \times 50 \mathrm{~mm}$ HPLC column (Waters, Milford, MA) with a mobile phase gradient.

For dextromethorphan and dextrorphan, a $50 \mu \mathrm{L}$ plasma sample was combined with $500 \mu \mathrm{L}$ of a deuterated internal standard (dextromethorphan-d3 and dextrorphan-d3 dissolved in methanol). After centrifugation, the supernatant was diluted with a reconstitution solution $(0.1 \%$ formic acid) and $10 \mu \mathrm{L}$ of the sample was injected to the LC-MS/MS system. Chromatographic separation was achieved on a Hypersil GOLD aQ $5 \mu \mathrm{m}, 4.6 \times 50 \mathrm{~mm}$ HPLC column (Thermo Scientific, Waltham, MA) with an isocratic condition.

For caffeine and paraxanthine, a $50 \mu \mathrm{L}$ plasma sample was combined with $50 \mu \mathrm{L}$ internal standard solution (caffeine-d3 dissolved in 50:50 methanol water) followed by addition of $1 \mathrm{~mL}$ methanol to precipitate the protein. After centrifugation, the supernatant was diluted $0.01 \mathrm{~N} \mathrm{HCl}$ and $10 \mu \mathrm{L}$ was injected to the LC-MS/MS system. The chromatographic separation was achieved on a YMCbasic $3 \mu \mathrm{m}, \mathrm{C}-8,4.6 \times 50 \mathrm{~mm}$ HPLC column (YMC America, Allentown, PA) with a mobile phase gradient.

The mass spectrometers were operated in positive electrospray ionization (ESI) mode. The selected reaction monitoring transitions were $346.2 \mathrm{~m} / \mathrm{z} \rightarrow 198.1 \mathrm{~m} / \mathrm{z}$ for omeprazole; $349.2 \mathrm{~m} / \mathrm{z} \rightarrow 198.1 \mathrm{~m} / \mathrm{z}$ for omeprazole-d3; $362.1 \mathrm{~m} / \mathrm{z} \rightarrow 213.9 \mathrm{~m} / \mathrm{z}$ for 5-hydroxyomeprazole; $365.1 \mathrm{~m} / \mathrm{z}$ $\rightarrow 213.9 \mathrm{~m} / \mathrm{z}$ for 5-hydroxyomeprazole-d3; $326.0 \mathrm{~m} / \mathrm{z}$ $\rightarrow 291.0 \mathrm{~m} / \mathrm{z}$ for midazolam; $330.0 \mathrm{~m} / \mathrm{z} \rightarrow 294.0 \mathrm{~m} / \mathrm{z}$ for the midazolam-d4; $342.0 \mathrm{~m} / \mathrm{z} \rightarrow 203.0 \mathrm{~m} / \mathrm{z}$ for $1^{\prime}$-hydroxymidazolam; $346.0 \mathrm{~m} / \mathrm{z} \rightarrow 203.0 \mathrm{~m} / \mathrm{z}$ for $\boldsymbol{\alpha}$-hydroxymidazolam-d4; $272.2 \mathrm{~m} / \mathrm{z} \rightarrow 171.0 \mathrm{~m} / \mathrm{z}$ for dextromethorphan; $275.2 \mathrm{~m} / \mathrm{z} \rightarrow 171.0 \mathrm{~m} / \mathrm{z}$ for dextromethorphan-d3; 258.2 $m / z \rightarrow 157.0 \mathrm{~m} / \mathrm{z}$ for dextrorphan, $261.2 \mathrm{~m} / \mathrm{z} \rightarrow 157.0$ $\mathrm{m} / \mathrm{z}$ for dextrorphan-d3; $195.1 \mathrm{~m} / \mathrm{z} \rightarrow 138.0 \mathrm{~m} / \mathrm{z}$ for caffeine; $181.0 \mathrm{~m} / \mathrm{z} \rightarrow 124.0 \mathrm{~m} / \mathrm{z}$ for paraxanthine; and $198.1 \mathrm{~m} / \mathrm{z} \rightarrow 138.0 \mathrm{~m} / \mathrm{z}$ for caffeine-d3.

Plasma concentrations were calculated using an 8-point curve with weighted linear regression. The nominal concentrations, based on standards that were prepared in human plasma, ranged from 1 to $100 \mathrm{ng} / \mathrm{mL}$ for omeprazole (USP, Rockville, MD, USA; Toronto Research Chemicals, Inc., Toronto, ON, Canada) and 5-hydroxyomeprazole (Toronto Research Chemicals, Inc., Toronto, ON, Canada),
0.1-100 ng/mL for midazolam (Cerilliant, Round Rock, TX, USA) and 1-hydroxymidazolam (Cerilliant, Round Rock, TX, USA; Lipomed, Cambridge, MA, USA), 0.05-50 ng/mL for dextromethorphan (USP, Rockville, MD, USA; Toronto Research Chemicals, Inc., Toronto, ON, Canada) and dextrorphan (TLC PharmaChem, Vaughan, ON, Canada; Cerilliant, Round Rock, TX, USA), and 20-20,000 ng/mL for caffeine (USP, Rockville, MD, USA; C/D/N Isotopes Inc., Pointe-Claire, QC, Canada) and paraxanthine (Toronto Research Chemicals, Inc., Toronto, ON, Canada). Lower limits of quantification were $1 \mathrm{ng} / \mathrm{mL}$ for omeprazole and 5-hydroxyomeprazole, $0.1 \mathrm{ng} / \mathrm{mL}$ for midazolam and 1-hydroxymidazolam, $0.05 \mathrm{ng} / \mathrm{mL}$ for dextromethorphan and dextrorphan, and $20 \mathrm{ng} / \mathrm{mL}$ for caffeine and paraxanthine. Quality-control samples (omeprazole and 5-hydroxyomeprazole: 3, 300, 750, and $7500 \mathrm{ng} / \mathrm{mL}$; midazolam and 1-hydroxymidazolam: 0.3 , 18, and $78 \mathrm{ng} / \mathrm{mL}$; dextromethorphan and dextrorphan: $0.15,4$, and $39 \mathrm{ng} / \mathrm{mL}$; caffeine and paraxanthine: 60, 7900 , and $15,800 \mathrm{ng} / \mathrm{mL}$ ) were prepared in separate batches and stored at $-20{ }^{\circ} \mathrm{C}$. Supplemental Table 1 (see electronic supplementary material) summarizes the performance characteristics of all the assays.

\subsubsection{Lisdexamfetamine and D-Amphetamine Analysis}

Concentrations of LDX and D-amphetamine were determined using a validated LC-MS/MS method. The LC-MS/MS system was a Sciex API-4000 mass spectrometer coupled with a Shimadzu HPLC system. The selected reaction monitoring transitions were $136.1 \rightarrow$ $119.1 \mathrm{~m} / \mathrm{z}$ for amphetamine, $141.1 \rightarrow 124.1 \mathrm{~m} / \mathrm{z}$, for amphetamine-d5 internal standard, $264.2 \rightarrow 84.2 \mathrm{~m} / \mathrm{z}$ for lisdexamfetamine, and $268.2 \rightarrow 88.2 \mathrm{~m} / \mathrm{z}$ for lisdexamfetamine$\mathrm{d} 4$ internal standard.

A $100-\mu \mathrm{L}$ plasma sample was combined with $50 \mu \mathrm{L}$ of the internal standard; proteins were precipitated by adding $500 \mu \mathrm{L}$ of a chilled acetonitrile:formic acid (100:5; volume to volume) solution. After vortexing and centrifugation, the supernatant was then added to $300 \mu \mathrm{L}$ of the reconstitution solution. Plasma concentrations of LDX and D-amphetamine were calculated using an 8-point curve with weighted linear regression. The nominal concentrations, based on standards that were prepared in human plasma, ranged from 1 to $100 \mathrm{ng} / \mathrm{mL}$ for LDX (Cerilliant, Round Rock, TX, USA; Alsachim, Illkirch-Graffenstaden, France) and from 2 to $200 \mathrm{ng} / \mathrm{mL}$ for D-amphetamine (Cerilliant, Round Rock, TX, USA). Lower limits of quantification were $1 \mathrm{ng} / \mathrm{mL}$ for LDX and $2 \mathrm{ng} / \mathrm{mL}$ for D-amphetamine. Quality-control samples for $\operatorname{LDX}(3,20,80$, and $100 \mathrm{ng} / \mathrm{mL})$ and D-amphetamine $(6,40,160$, and $200 \mathrm{ng} / \mathrm{mL}$ ) were prepared and stored at $-20{ }^{\circ} \mathrm{C}$ before the start of the analysis. Supplemental Table 1 (see electronic 
supplementary material) summarizes the performance characteristics of the assays.

\subsection{Safety and Tolerability Endpoints}

Safety and tolerability endpoints included assessments of vital signs, 12-lead ECGs, physical examinations, clinical safety laboratory tests, and AEs. Participant-reported and investigator-observed AEs were recorded at screening, on each day during the two treatment periods, and at followup. AEs were classified according to severity and their relationship to the study drug. Vital signs were assessed at screening, on day -1 of each treatment period, at $30 \mathrm{~min}$ pre-dose and 2, 4, 8, and $12 \mathrm{~h}$ post-dose on day 1 of each period, and on days 2 (i.e., $24 \mathrm{~h}$ post-dose), 3 (i.e., $48 \mathrm{~h}$ post-dose), and 4 (i.e., $72 \mathrm{~h}$ post-dose) of each treatment period. Physical and clinical laboratory examinations were assessed at screening, on day -1 of each treatment period, and on day 4 of period 2; ECGs were assessed at screening, on days -1 and 1 of each treatment period, and on day 4 of period 2.

\subsection{Data Presentation and Statistical Analyses}

The sample size was estimated to determine equivalence between the two regimens for each pharmacokinetic parameter for each substrate in the Cooperstown cocktail. For a true mean ratio of 0.95 , it was estimated that 24 participants needed to complete the study to achieve $85 \%$ power and a 1-sided $\alpha$ of 0.05 (corresponding to $90 \% \mathrm{CI}$ ).

All pharmacokinetic analyses were conducted using WinNonlin Phoenix version 6.3 or higher (Pharsight Corp., Mountain View, CA) in the pharmacokinetic analysis set, which consisted of all participants in the safety analysis set for whom the primary pharmacokinetic data were considered sufficient and interpretable. Analysis of variance, with period and treatment regimen as fixed effects and participant nested within sequence as a random effect, was used to compare the means of log-transformed $C_{\max }$ and $\mathrm{AUC}_{0-\infty}$ between treatment regimens for each analyte. To assess treatment effect magnitude for $C_{\max }$ and $\mathrm{AUC}_{0-\infty}$, geometric mean ratios and $90 \%$ CIs were calculated. Consistent with guidelines of the Food and Drug Administration for drug-drug interaction studies [14], bioequivalence test rules were utilized to determine if the $90 \%$ CI of the geometric mean ratio was within the interval of $0.80-1.25$. Treatments were considered equivalent, and thus no interaction was concluded, if the $90 \% \mathrm{CI}$ of the geometric mean ratio was within the interval of 0.80-1.25. If the $90 \%$ CI was not wholly contained within the interval of $0.80-1.25$, equivalence was not concluded and a drug interaction could not be excluded. Descriptive statistics were also determined for all pharmacokinetic parameters for each analyte under both treatment regimens. The safety analysis set included participants who received one or more doses of study drug and had one or more post-dose safety assessments. Safety endpoints were summarized using descriptive statistics.

\section{Results}

\subsection{Participant Disposition and Demographics}

A total of 30 participants were enrolled and randomized (15 participants were randomized to receive Cooperstown cocktail as the initial treatment; 15 were randomized to receive Cooperstown cocktail + LDX as the initial treatment); 29 participants completed the study. All 30 participants (15 per group) were included in the randomized, pharmacokinetic, and safety analysis sets; one participant who received the Cooperstown cocktail as the initial treatment discontinued before completing the study (primary reason: "withdrawal by participant").

Overall, the mean $\pm \mathrm{SD}$ age of participants was $35.6 \pm 7.91$ years; the majority were White $(26 / 30$; $86.7 \%)$ and men $(18 / 30 ; 60.0 \%)$. Participant demographics by treatment regimen are summarized in Supplemental Table 2 (see electronic supplementary material).

\subsection{Pharmacokinetic Endpoints}

Figure 1 depicts the mean \pm SD plasma concentrations over time by treatment regimen for the parent substrates and their primary metabolites. Table 1 presents descriptive statistics for pharmacokinetic parameters by treatment regimen for each CYP substrate, its respective metabolite, and LDX and D-amphetamine.

Geometric least squares (LS) means for $C_{\max }$ and $\mathrm{AUC}_{0-\infty}$ for each treatment and the ratios between treatments are summarized in Table 2. The geometric LS mean ratios for $\mathrm{AUC}_{0-\infty}$ for all treatments were fully contained within the $80-125 \%$ range. Geometric LS mean ratios for $C_{\max }$ for midazolam, caffeine, and their metabolites were also fully contained within the $80-125 \%$ range. The upper $90 \% \mathrm{CI}$ for the $C_{\max }$ geometric LS mean ratio was outside the 80-125\% range for dextromethorphan, but the $90 \%$ CIs were within the accepted range for dextrorphan. For omeprazole and 5-hydroxyomeprazole, the geometric LS mean ratios were below the lower limit of the 80-125\% range.

The mean \pm SD parent to metabolite ratios for $C_{\max }$ and $\mathrm{AUC}_{0-\infty}$ were similar between treatments (Table 2), with the exception of dextromethorphan to dextrorphan for $\mathrm{AUC}_{0-\infty}$, which was lower when LDX was administered with the Cooperstown cocktail. Upon further examination 

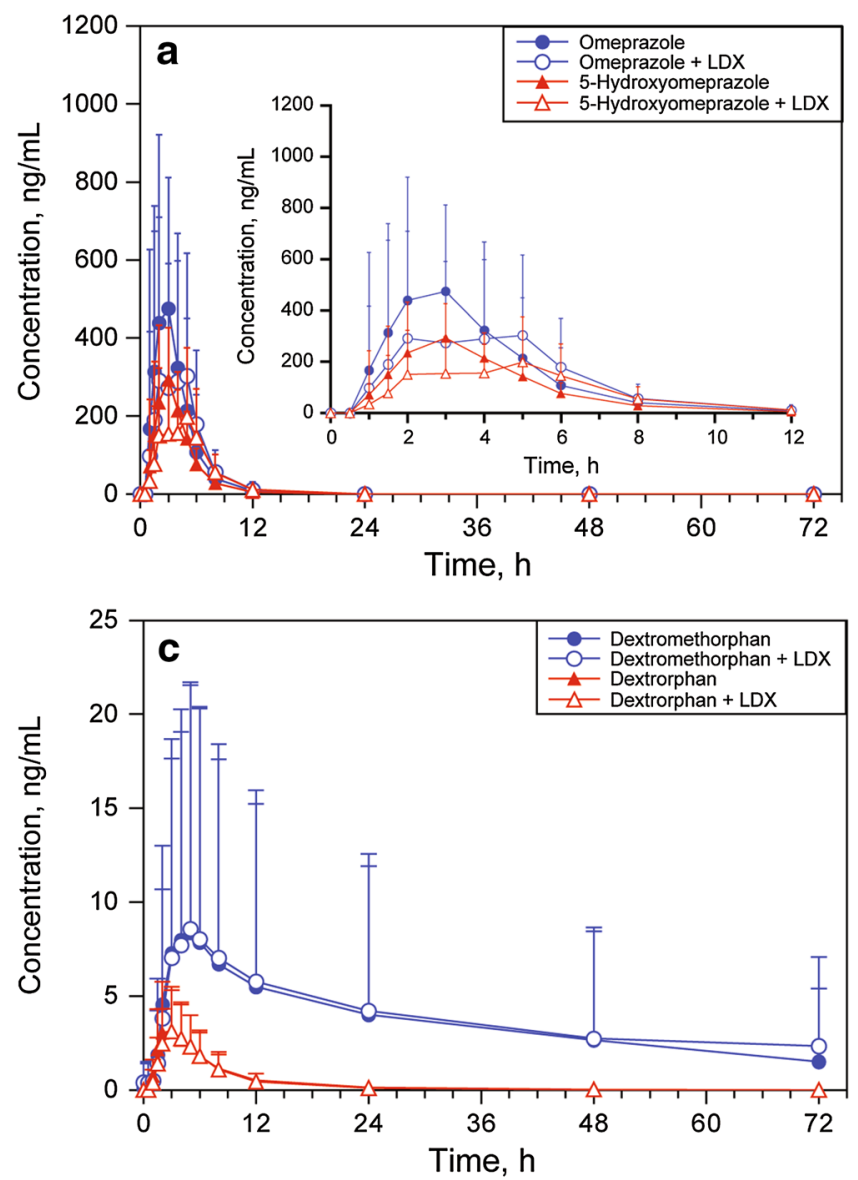

Fig. 1 Mean \pm SD plasma concentrations for a omeprazole and 5-hydroxyomeprazole, b midazolam and 1-hydroxymidazolam, c dextromethorphan and dextrorphan, and $\mathbf{d}$ caffeine and paraxanthine by treatment regimen, pharmacokinetic analysis set. Lower limits of

of the dextromethorphan to dextrorphan ratio, it was observed that five participants had substantially higher ratios for both $C_{\max }$ and $\mathrm{AUC}_{0-\infty}$, which skewed the mean values for these parameters. Between treatment regimens, geometric mean values for the dextromethorphan to dextrorphan ratio for $C_{\max }$ (Cooperstown cocktail alone: 0.9588; Cooperstown cocktail + LDX: 1.2521) and $\mathrm{AUC}_{0-\infty}$ (Cooperstown cocktail alone: 1.7023; Cooperstown cocktail + LDX: 1.8868) were similar.

\subsection{Safety and Tolerability Endpoints}

The frequency of treatment-emergent AEs (TEAEs) is summarized in Supplemental Table 3 (see electronic supplementary material). All TEAEs were mild in severity and considered related to the study drug (any TEAEs, $n[\%]$ : Cooperstown cocktail alone, 18 [60.0]; Cooperstown cocktail + LDX, 26 [89.7]). There were no serious or severe TEAEs, no discontinuations due to TEAEs, and no deaths reported during the study. Supplemental Table 3
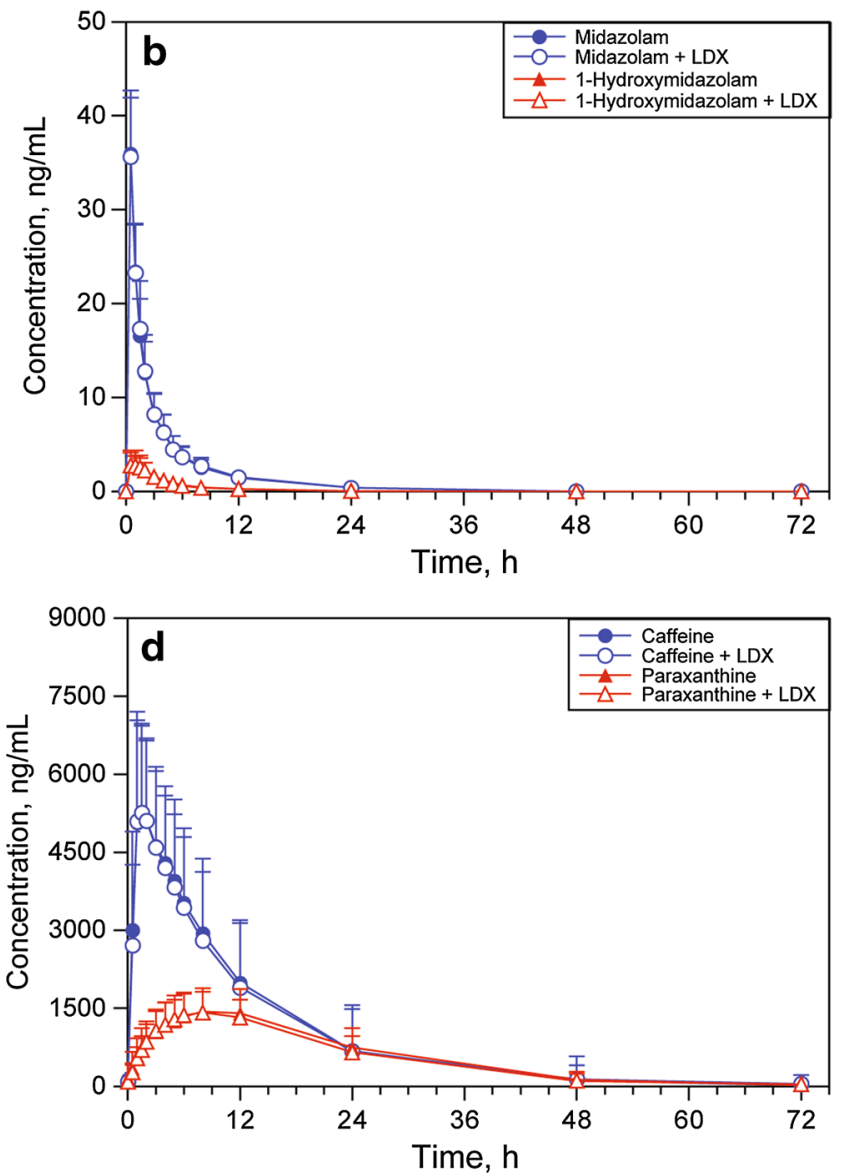

quantification: omeprazole and 5-hydroxyomeprazole $(1 \mathrm{ng} / \mathrm{mL})$, midazolam and 1-hydroxymidazolam $(0.1 \mathrm{ng} / \mathrm{mL})$, dextromethorphan and dextrorphan $(0.05 \mathrm{ng} / \mathrm{mL})$, caffeine and paraxanthine (20 ng/mL). LDX lisdexamfetamine dimesylate

(see electronic supplementary material) shows the frequency of TEAEs reported by $\geq 5 \%$ of participants in either treatment group. With the Cooperstown cocktail alone, the most frequent TEAEs ( $n[\%])$ were somnolence $(13$ [43.3]) and dizziness (4 [13.3]); with the Cooperstown cocktail + LDX, the most frequent TEAEs included insomnia (12 [41.4]), somnolence (10 [34.5]), dry mouth (9 [31.0]), increased blood pressure (8 [27.6]), tachycardia and dizziness (both 5 [17.2]), decreased appetite and euphoric mood (both 4 [13.8]), nausea, anxiety, and depressed mood (each 3 [10.3]).

Supplemental Table 4 (see electronic supplementary material) summarizes mean \pm SD changes from baseline for vital sign assessments. SBP, DBP, and pulse rate increased more with the Cooperstown cocktail + LDX compared with the Cooperstown cocktail alone at 4, 8, and $12 \mathrm{~h}$ post-dose. Mean \pm SD SBP (Cooperstown cocktail alone: $2.6 \pm 7.76$; Cooperstown cocktail + LDX: $16.9 \pm 12.57$ ) and DBP (Cooperstown cocktail alone: $1.8 \pm 7.71$; Cooperstown cocktail + LDX: $10.6 \pm 10.33$ ) 


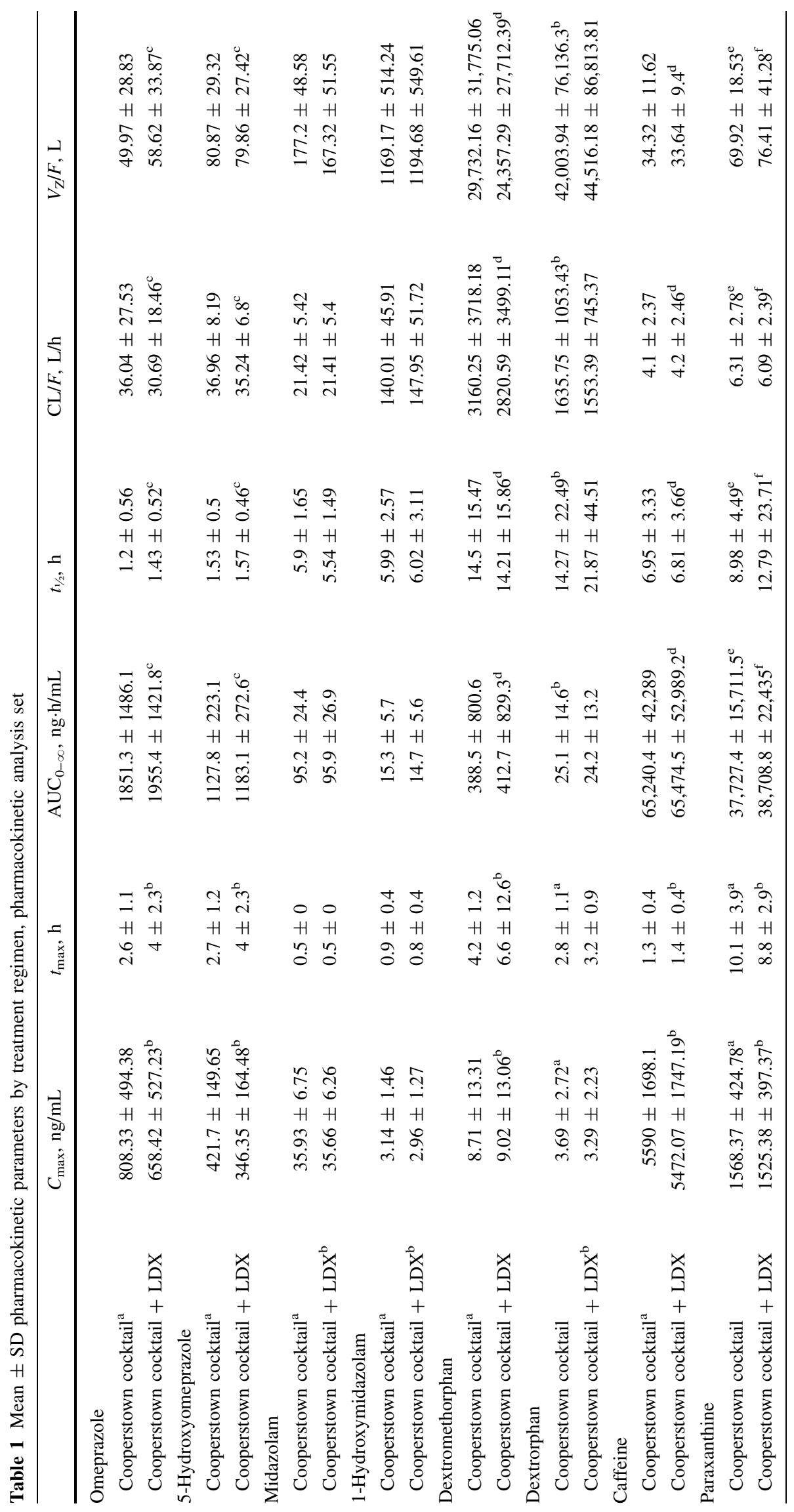




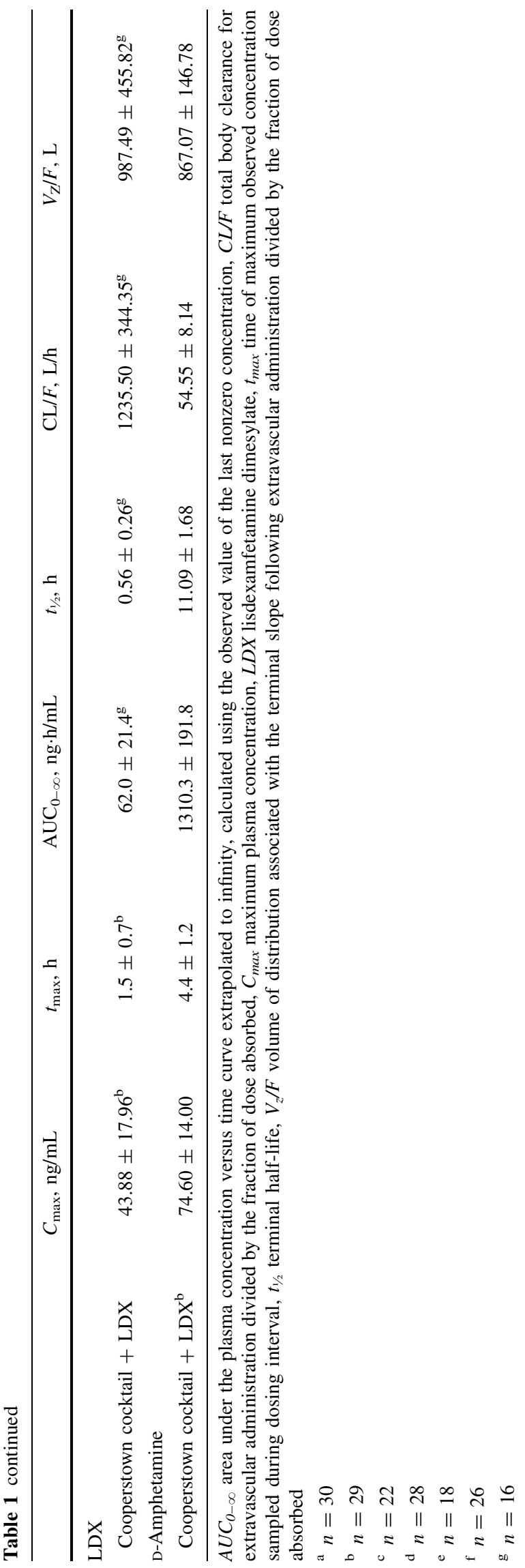

changes from baseline peaked at $8 \mathrm{~h}$ post-dose; changes were similar between treatments at $24 \mathrm{~h}$ post-dose. Mean \pm SD increases from baseline for pulse rate peaked at $12 \mathrm{~h}$ post-dose (Cooperstown cocktail alone: $3.7 \pm 8.37$; Cooperstown cocktail + LDX: $23.1 \pm 14.63)$ and remained higher at $24 \mathrm{~h}$ post-dose with the Cooperstown cocktail + LDX $(9.0 \pm 12.18)$ compared with the Cooperstown cocktail alone $(4.5 \pm 10.89)$. Mean \pm SD changes from baseline at day 4 were small for heart rate (Cooperstown cocktail, $5.3 \pm 6.36$; Cooperstown cocktail + LDX, $4.9 \pm 12.60)$. No baseline or post-baseline ECG evaluations were considered clinically relevant.

\section{Discussion}

This is the first study to simultaneously assess the effects of LDX on the activity of multiple CYP enzymes in healthy human volunteers. The results suggest that LDX does not alter the activity of the CYP1A2, CYP2D6, or CYP3A enzymes, as evidenced by a lack of LDX effect on $\mathrm{AUC}_{0-\infty}$ for substrates of these enzymes. As such, LDX is not expected to alter the pharmacokinetics of concomitantly administered medications that require CYP1A2, CYP2D6, or CYP3A enzyme activity for their metabolism. Additionally, coadministration of the Cooperstown cocktail with LDX did not alter LDX or amphetamine exposure, as the pharmacokinetic profiles for LDX and D-amphetamine observed in this study were similar to those observed in other single-dose LDX studies in healthy adults [15, 16].

These findings are consistent with previously reported data from an in vitro study, which reported that incubation of LDX with human liver microsomes was not associated with concentration-dependent or mechanism-based inhibition of various CYP enzymes [9]. The data are also generally consistent with findings in healthy volunteers. Coadministration of LDX and GXR, which is metabolized by CYP3A4 [12], produced only small increases in $C_{\max }$ for GXR in one study [11]. In another study, coadministration of LDX with VXR, which is metabolized by CYP2D6 and CYP3A4 [4], was associated with only minor increases in VXR exposure, minor decreases in exposure to ODV, and no change in VXR + ODV exposure when compared with VXR alone in healthy volunteers [10].

A small reduction in $C_{\max }$ for omeprazole and its metabolite, 5-hydroxyomeprazole, was observed in this study. This reduction could be the result of changes in CYP2C19 activity or an effect of LDX on the absorption of omeprazole. However, the latter is the more likely explanation because the conversion rate from omeprazole to 5-hydroxyomeprazole was unaffected by LDX, as evidenced by the omeprazole to 5-hydroxyomeprazole ratio and the lack of change in total exposure based on $\mathrm{AUC}_{0-\infty}$. 
Table 2 Geometric LS means and ratios for parent compounds and metabolites, and mean parent to metabolite ratios for $C_{\max }$ and $\mathrm{AUC}_{0-\infty}$ by treatment regimen, pharmacokinetic analysis set

\begin{tabular}{|c|c|c|c|c|}
\hline & \multicolumn{2}{|c|}{$C_{\max }, \mathrm{ng} / \mathrm{mL}$} & \multicolumn{2}{|c|}{$\mathrm{AUC}_{0-\infty}, \mathrm{ng} \cdot \mathrm{h} / \mathrm{mL}$} \\
\hline & $\begin{array}{l}\text { Geometric } \\
\text { LS mean }\end{array}$ & $\begin{array}{l}\text { Cooperstown cocktail + LDX/ } \\
\text { Cooperstown cocktail }(90 \% \mathrm{CI}) \text { ratio }\end{array}$ & $\begin{array}{l}\text { Geometric } \\
\text { LS mean }\end{array}$ & $\begin{array}{l}\text { Cooperstown cocktail + LDX/ } \\
\text { Cooperstown cocktail }(90 \% \mathrm{CI}) \text { ratio }\end{array}$ \\
\hline \multicolumn{5}{|l|}{ Omeprazole } \\
\hline $\begin{array}{l}\text { Cooperstown } \\
\text { cocktail }\end{array}$ & 677.9 & $0.689(0.527-0.9)$ & 1428 & $1.049(0.99-1.112)$ \\
\hline $\begin{array}{l}\text { Cooperstown } \\
\text { cocktail + LDX }\end{array}$ & 466.9 & & 1499 & \\
\hline \multicolumn{5}{|c|}{ 5-Hydroxyomeprazole } \\
\hline $\begin{array}{l}\text { Cooperstown } \\
\text { cocktail }\end{array}$ & 396.5 & $0.732(0.596-0.899)$ & 1106 & 1.009 (0.975-1.045) \\
\hline $\begin{array}{l}\text { Cooperstown } \\
\text { cocktail + LDX }\end{array}$ & 290.2 & & 1116 & \\
\hline \multicolumn{5}{|l|}{ Midazolam } \\
\hline $\begin{array}{l}\text { Cooperstown } \\
\text { cocktail }\end{array}$ & 35.23 & $0.996(0.952-1.043)$ & 92.07 & $1.011(0.978-1.044)$ \\
\hline $\begin{array}{l}\text { Cooperstown } \\
\text { cocktail + LDX }\end{array}$ & 35.11 & & 93.04 & \\
\hline \multicolumn{5}{|c|}{ 1-Hydroxymidazolam } \\
\hline $\begin{array}{l}\text { Cooperstown } \\
\text { cocktail }\end{array}$ & 2.89 & $0.953(0.888-1.022)$ & 14.41 & $0.955(0.916-0.997)$ \\
\hline $\begin{array}{l}\text { Cooperstown } \\
\text { cocktail + LDX }\end{array}$ & 2.75 & & 13.77 & \\
\hline \multicolumn{5}{|l|}{ Dextromethorphan } \\
\hline $\begin{array}{l}\text { Cooperstown } \\
\text { cocktail }\end{array}$ & 2.43 & $1.181(1.007-1.384)$ & 34.85 & $1.069(0.965-1.185)$ \\
\hline $\begin{array}{l}\text { Cooperstown } \\
\text { cocktail + LDX }\end{array}$ & 2.87 & & 37.27 & \\
\hline \multicolumn{5}{|l|}{ Dextrorphan } \\
\hline $\begin{array}{l}\text { Cooperstown } \\
\text { cocktail }\end{array}$ & 2.54 & $0.938(0.849-1.037)$ & 21.47 & $1.016(0.913-1.129)$ \\
\hline $\begin{array}{l}\text { Cooperstown } \\
\text { cocktail + LDX }\end{array}$ & 2.38 & & 21.80 & \\
\hline \multicolumn{5}{|l|}{ Caffeine } \\
\hline $\begin{array}{l}\text { Cooperstown } \\
\text { cocktail }\end{array}$ & 5370 & $0.977(0.945-1.01)$ & 56,207 & $1.009(0.959-1.06)$ \\
\hline $\begin{array}{l}\text { Cooperstown } \\
\text { cocktail + LDX }\end{array}$ & 5246 & & 56,688 & \\
\hline \multicolumn{5}{|l|}{ Paraxanthine } \\
\hline $\begin{array}{l}\text { Cooperstown } \\
\text { cocktail }\end{array}$ & 1520 & $0.969(0.924-1.016)$ & 36,380 & $0.984(0.91-1.065)$ \\
\hline $\begin{array}{l}\text { Cooperstown } \\
\text { cocktail + LDX }\end{array}$ & 1473 & & 35,809 & \\
\hline
\end{tabular}

Omeprazole to 5-hydroxyomeprazole ratio

Cooperstown cocktail ${ }^{\mathrm{a}}$

Cooperstown cocktail + LDX

Midazolam to 1-hydroxymidazolam ratio
Cooperstown cocktail ${ }^{\mathrm{a}}$
Cooperstown cocktail $+\mathrm{LDX}^{\mathrm{b}}$

$$
\begin{aligned}
2.0779 & \pm 1.4317 \\
1.8809 & \pm 1.3421^{\mathrm{b}} \\
13.1254 & \pm 5.2713 \\
13.8779 & \pm 6.0046
\end{aligned}
$$$$
1.6082 \pm 1.2363
$$$$
1.7008 \pm 1.3556^{\mathrm{c}}
$$$$
6.9056 \pm 2.7659
$$ 
Table 2 continued

\begin{tabular}{lcc}
\hline & Mean $\pm \mathrm{SD} C_{\max }, \mathrm{ng} / \mathrm{mL}$ & $\mathrm{Mean} \pm \mathrm{SD} \mathrm{AUC} \mathrm{C}_{0-\infty}, \mathrm{ng} \cdot \mathrm{h} / \mathrm{mL}$ \\
\hline Dextromethorphan to dextrorphan ratio & & $34.3259 \pm 82.8729^{\mathrm{b}}$ \\
Cooperstown cocktail & $19.6953 \pm 45.9325^{\mathrm{a}}$ & $21.8684 \pm 47.5228^{\mathrm{d}}$ \\
Cooperstown cocktail + LDX & $18.9546 \pm 43.0244^{\mathrm{b}}$ & $1.5256 \pm 0.3902^{\mathrm{e}}$ \\
Caffeine to paraxanthine ratio & & $1.6297 \pm 0.3875^{\mathrm{f}}$ \\
Cooperstown cocktail & $3.6489 \pm 1.1012^{\mathrm{a}}$ & $3.75 \pm 1.7019^{\mathrm{b}}$ \\
Cooperstown cocktail + LDX & & \\
\hline
\end{tabular}

$A U C_{0-\infty}$ area under the plasma concentration versus time curve extrapolated to infinity, calculated using the observed value of the last nonzero concentration, $C_{\max }$ maximum plasma concentration, $L D X$ lisdexamfetamine dimesylate, $L S$ least squares

a $n=30$

b $n=29$

c $n=22$

d $n=28$

e $n=18$

${ }^{\mathrm{f}} n=26$

This interpretation is supported by preclinical in vitro data that reported that LDX was not associated with changes in CYP2C19 activity [9]. Although a previously published study in healthy adults reported that combined administration of omeprazole with LDX or mixed amphetamine salts-extended release (MAS-XR) did not significantly alter total amphetamine exposure from LDX or MAS-XR [17], omeprazole pharmacokinetic parameters were not assessed in this earlier study. Therefore, it is not known whether coadministration of LDX + omeprazole was associated with alterations in omeprazole pharmacokinetic parameters in that study.

The pattern of TEAEs with LDX (i.e., insomnia, dry mouth, increased blood pressure, and tachycardia) in this study was generally consistent with a previously reported LDX profile in healthy volunteers [10]. No serious or severe TEAEs or study discontinuations due to TEAEs were reported in this study. Changes in vital signs and ECG assessments were also generally consistent with other studies in healthy adults $[10,15]$.

Several limitations of this study should be noted in regard to interpretation of these findings. First, it is important to note that only single doses of LDX and each CYP substrate in the Cooperstown cocktail were used in this study. As such, it is unclear if the use of higher doses of the CYP substrates, which are more likely to saturate CYP systems, may alter these findings or if repeated dosing would influence the results. Third, this study did not assess the effects of strong CYP inhibitors on the pharmacokinetic profile of LDX, so it is unclear how coadministration of these compounds would influence the pharmacokinetics of LDX. However, no changes in LDX or D-amphetamine exposure were observed in healthy volunteers after the coadministration of VXR, which is not a typical strong inhibitor of CYP2D6 but is metabolized by CYP2D6 [4] and LDX [10]. Finally, findings related to safety and tolerability from this phase I study should be interpreted with caution because of the small sample size.

\section{Conclusions}

In summary, at the doses tested in this study, LDX did not alter the activity of the CYP1A2, CYP2D6, or CYP3A enzymes in healthy volunteers. This suggests that drug interactions with medications that are metabolized by these enzymes would not be expected when administered in conjunction with LDX. This is of particular importance in situations where individuals with ADHD are treated for comorbid conditions, including psychiatric disorders. A small $C_{\max }$ reduction for omeprazole and its metabolite was observed when LDX was administered with the Cooperstown cocktail, which may be an effect on absorption of omeprazole. Although an effect of LDX on CYP2C19 is likely to be minimal, further investigation may be warranted to more fully explore the impact of LDX on CYP2C19 activity.

Acknowledgments and conflicts of interest Shire Development LLC (Wayne, PA) provided funding to Complete Healthcare Communications, Inc. (CHC; Chadds Ford, PA) for support in writing and editing this manuscript. Under the direction of the authors, Stefan Kolata, PhD, and Craig Slawecki, PhD, full-time employees of Complete Healthcare Communications (Chadds Ford, PA), provided writing and formatting assistance for this manuscript. Brian Scheckner, PharmD, and Phillip Wang, PhD, MBA, of Shire also reviewed and edited the manuscript for scientific accuracy. James Ermer, Mary Corcoran, and Patrick Martin are employees of Shire and hold stock and/or options in Shire. 
Ethical standards The protocol was approved by the institutional review board of the study site before the initiation of the study, and the study was conducted in accordance with the International Conference on Harmonisation and Good Clinical Practice and with the Declaration of Helsinki.

Open Access This article is distributed under the terms of the Creative Commons Attribution Noncommercial License which permits any noncommercial use, distribution, and reproduction in any medium, provided the original author(s) and the source are credited.

\section{References}

1. McGough JJ, Smalley SL, McCracken JT, Yang M, Del'Homme M, Lynn DE, et al. Psychiatric comorbidity in adult attention deficit hyperactivity disorder: findings from multiplex families. Am J Psychiatry. 2005;162(9):1621-7.

2. Smalley SL, McGough JJ, Moilanen IK, Loo SK, Taanila A, Ebeling $\mathrm{H}$, et al. Prevalence and psychiatric comorbidity of attention-deficit/hyperactivity disorder in an adolescent Finnish population. J Am Acad Child Adolesc Psychiatry. 2007;46(12): 1575-83.

3. Sobanski E. Psychiatric comorbidity in adults with attentiondeficit/hyperactivity disorder (ADHD). Eur Arch Psychiatry Clin Neurosci. 2006;256(Suppl. 1):i26-31.

4. Spina E, Santoro V, D'Arrigo C. Clinically relevant pharmacokinetic drug interactions with second-generation antidepressants: an update. Clin Ther. 2008;30(7):1206-27.

5. Hodgkins P, Shaw M, McCarthy S, Sallee FR. The pharmacology and clinical outcomes of amphetamines to treat ADHD: does composition matter? CNS Drugs. 2012;26(3):245-68.

6. Bach MV, Coutts RT, Baker GB. Involvement of CYP2D6 in the in vitro metabolism of amphetamine, two $N$-alkylamphetamines and their 4-methoxylated derivatives. Xenobiotica. 1999;29(7): 719-32.

7. Pennick M. Absorption of lisdexamfetamine dimesylate and its enzymatic conversion to D-amphetamine. Neuropsychiatr Dis Treat. 2010;6:317-27.
8. Vyvanse ${ }^{\circledR}$ (lisdexamfetamine dimesylate). Full Prescribing Information, Shire US Inc., Wayne, PA, 2015.

9. Krishnan S, Moncrief S. An evaluation of the cytochrome P450 inhibition potential of lisdexamfetamine in human liver microsomes. Drug Metab Dispos. 2007;35(1):180-4.

10. Ermer J, Haffey MB, Richards C, Lasseter K, Roesch B, Purkayastha $\mathrm{J}$, et al. An open-label investigation of the pharmacokinetic profiles of lisdexamfetamine dimesylate and venlafaxine extended-release, administered alone and in combination, in healthy adults. Clin Drug Investig. 2013;33(4):243-54.

11. Roesch B, Corcoran ME, Fetterolf J, Haffey M, Martin P, Preston $\mathrm{P}$, et al. Pharmacokinetics of coadministered guanfacine extended release and lisdexamfetamine dimesylate. Drugs R D. 2013; 13(2):119-28.

12. Caley CF. Interpreting and applying CYP450 genomic test results to psychotropic medications. J Pharm Pract. 2011;24(5):439-46.

13. Streetman DS, Bleakley JF, Kim JS, Nafziger AN, Leeder JS, Gaedigk A, et al. Combined phenotypic assessment of CYP1A2, CYP2C19, CYP2D6, CYP3A, $N$-acetyltransferase-2, and xanthine oxidase with the "Cooperstown cocktail". Clin Pharmacol Ther. 2000;68(4):375-83.

14. US Food and Drug Administration. Guidance for industry: drug interaction studies - study design, data analysis, implications for dosing, and labeling recommendations. Rockville: US Food and Drug Administration, Center for Drug Evaluation and Research (CDER); 2012.

15. Krishnan S, Zhang Y. Relative bioavailability of lisdexamfetamine 70-mg capsules in fasted and fed healthy adult volunteers and in solution: a single-dose, crossover pharmacokinetic study. J Clin Pharmacol. 2008;48(3):293-302.

16. Krishnan SM, Pennick M, Stark JG. Metabolism, distribution and elimination of lisdexamfetamine dimesylate: open-label, singlecentre, phase I study in healthy adult volunteers. Clin Drug Investig. 2008;28(12):745-55.

17. Haffey MB, Buckwalter M, Zhang P, Homolka R, Martin P, Lasseter KC, et al. Effects of omeprazole on the pharmacokinetic profiles of lisdexamfetamine dimesylate and extended-release mixed amphetamine salts in adults. Postgrad Med. 2009;121(5): $11-9$. 